\title{
Knowledge, Attitude and Practice of Kangaroo Mother Care by Postnatal Mothers who Gave Birth to Preterm and Low Birth Weight Babies in Public Hospitals, Eastern Ethiopia
}

\section{Aklilu Abrham Roba*, Susan Binoy and Mahantesh A Naganuri}

College of Health and Medical Sciences, Haramaya University, Ethiopia

*Corresponding author: Aklilu Abrham Roba, Department of Paediatrics and Child Health Nursing, College of Health and Medical Sciences, Haramaya University, Ethiopia, Tel: +251923276062; E-mail: akliltimnathserah@gmail.com

Received date: October 25, 2017; Accepted date: November 20, 2017; Published date: November 27, 2017

Copyright: (c) 2017 Roba AA, et al. This is an open-access article distributed under the terms of the Creative Commons Attribution License, which permits unrestricted use, distribution, and reproduction in any medium, provided the original author and source are credited.

\begin{abstract}
Introduction: Globally, babies born with low birth weight and preterm accounts for 25 million and 15 million respectively. The magnitude of preterm and low birth weight was $29.1 \%$ in Ethiopia by 2011 . Around $70 \%$ neonatal deaths occur in low birth weight and preterm babies. Most studies demonstrated kangaroo mother care to be more effective than incubator care for these neonates but there are limited studies in Ethiopia. Therefore, the main aim of the study was to assess the acceptability, knowledge, attitude and practice of kangaroo mother care in public hospitals.
\end{abstract}

Methods: A descriptive cross-sectional study was conducted among postnatal mothers of preterm and low birth weight babies in Dilchora and Hiwot Fana Specialized hospital from October 1, 2015 to June 25, 2016. Data were collected by interviewing 349 mothers by a pretested questionnaire. It was entered in to Epi Data software version 3.1 and exported to SPSS version 20 for analysis.

Results: $69.91 \%$ of mothers mentioned the benefits of kangaroo mother care correctly. The majority of mothers $221(63.33 \%)$ felt positive regarding implementation of Kangaroo Mother Care for it corrects the temperature, increase attachment and improve the growth of their small babies. On the other hand, $195(55.87 \%)$ believed that Kangaroo Mother Care has positive effect on breast feeding. During the study period, $189(54.15 \%)$ mothers practiced Kangaroo Mother Care in hospitals and also willing to continue at home. The mean duration of Kangaroo Mother Care was $2 \mathrm{~h} /$ day.

Conclusion: The majority of mothers felt positive regarding implementation of Kangaroo Mother Care. The study also revealed that more than half of the study participants practiced Kangaroo Mother Care in hospitals and also willing to continue at home. Therefore, it will be crucial if there are health education sessions during antenatal care follow-up for complete acceptance of Kangaroo Mother Care after delivery.

Keywords: Kangaroo mother care; Preterm and low birth weight; Small babies; Ethiopia

\section{Introduction}

Globally, babies with low birth weight, and preterm accounts 25 and 15 million, respectively. Almost all of them (96\%) are in developing countries. The rates are highest in Africa which has $12 \%$ of the world's population but over $25 \%$ of the world's new-born deaths [1,2]. Almost 1 million children die due to complications of preterm birth. Low birth weight refers to birth weight below $2500 \mathrm{~g}$ [3]. Preterm is defined as babies born alive before 37 completed weeks of gestation. There are three sub-categories of preterm based on gestational age: Extremely preterm $(<28$ weeks), Very preterm $(28$ to $<32$ weeks $)$ and moderate to late preterm (32 to $<37$ weeks) [4]. Low birth weight and prematurity are strongly associated with neonatal morbidities and mortality [2]. Neonatal hypothermia is an important challenge associated with morbidity and mortality [5].

In, Ethiopia, the prevalence of low birth weight varies from place to place. It was reported $28.3 \%$ in Kersa district, Oromia region, eastern
Ethiopia [6], and 22.5\% in Jimma zone, Southwest Ethiopia [7], 17.4\% in Gonder, northern Ethiopia [8]. On the other hand, in-depth analysis of the Ethiopian Demographic and Health survey 2011 indicated that $29.1 \%$ of Ethiopian babies were reported small at birth [9]. Caring for LBW infants' impose heavy burden on developing countries. World Health Organization (WHO) has recommended scaling up low cost solutions that could reduce these deaths by three-quarters which includes Kangaroo Mother Care (KMC) as one of the measures [1].

Kangaroo Mother Care is defined as a method of holding a small nappy neonate in skin-to skin contact (STS), prone and upright on the maternal chest. The neonate is enclosed in maternal clothing in order to maintain temperature stability [10]. It was invented by Dr. Rey in 1978 and developed by Dr. Martinez and Dr. Navarrete until 1994, when the Kangaroo Foundation was created. Initially KMC was developed as response to overcrowding, and insufficient resources in neonatal intensive care units. But today KMC is formally endorsed by WHO [11].

KMC has a lot of benefits to the mother, for the preterm and low birth weight infants, for the institution, and for the community at large. For mothers, it increases milk volume, double rates of successful 
Citation: Roba AA, Binoy S, Naganuri MA (2017) Knowledge, Attitude and Practice of Kangaroo Mother Care by Postnatal Mothers who Gave Birth to Preterm and Low Birth Weight Babies in Public Hospitals, Eastern Ethiopia. J Neonatal Biol 6: 264. doi: $10.4172 / 2167-0897.1000264$

Page 2 of 5

breastfeeding, feelings of confidence, competence, and satisfaction regarding baby care. For preterm and LBW infant, it normalizes temperature, heart rate, respiratory rate, and strengthens the infant's immune system. It also reduces physiologic and behavioural pain responses, increases weight gain and enhances mother-infant bonding. Furthermore it has Positive effects on infant's cognitive development, less nosocomial infection, and earlier discharge [12]. For institutions it shorten hospital stay, more parental involvement with greater opportunities for teaching and assessing, and better use of healthcare budgets. For the community it decreases morbidity and mortality especially in developing countries, and opportunities for teaching during pregnancy and follow-up in preparation for postnatal implementation, decreased use of financial resource and Promotion of total family health [12].

This innovative strategy (KMC) is being implemented in very few hospitals in Ethiopia. There is also limited data regarding KAP of KMC among postnatal mothers in Ethiopia in general and east Ethiopia in particular. So, this study was intended to investigate knowledge, attitude, practice and acceptance of kangaroo mother care of mothers in selected public hospitals in eastern Ethiopia.

\section{Materials and Methods}

\section{Study design, area and period}

The Institution based descriptive cross-sectional study was conducted in Hiwot Fana Specialized hospital (HFSH) in Harar and Dichora hospital in Dire Dawa, eastern Ethiopia. Harar is located 526 $\mathrm{km}$ away from the capital, Addis Ababa in the eastern direction. It is one of the most popular historical towns with a projected total population of 203,438 (M: $F=102,369: 101,069$ ) in 2010. Dire Dawa is located $515 \mathrm{~km}$ from Addis Ababa on the Addis Ababa-Djibouti railroad in the eastern part of Ethiopia. Based on the 2007 Census conducted by the Central Statistical Agency of Ethiopia (CSA), it had 341,834 populations; of whom 171,461 are men and 170,461 women. Dil Chora Referral Hospital (DCRH), the only governmental hospital in the region which was established in 1952. Since then, it has been serving the ever increasing population of the Dire Dawa City and its adjacent regions of Oromiya and Somali.

The study was conducted from October 1, 2015 to June 25, 2016.

\section{Sample size determination}

The sample size was calculated by using single population proportion at a precision of 5\%, 95\% confidence interval and prevalence of small babies 9LBW and preterm) $29.1 \%$ with $\mathrm{P}$ value of 0.291 [9], hence adding $10 \%$ non-response rate, the total sample size calculated was 349 postnatal mothers who gave birth to preterm and low birth weight.

\section{Sampling techniques}

Dilchora and Hiwot Fana Specialized hospitals were selected purposively due to advanced service they are providing including Neonatal Intensive Care Unit (NICU) and KMC units. Study participants were proportionally selected from both hospitals according to patient flow. Postnatal mothers that gave preterm and/or LBW were selected consecutively until the allocated sample size was obtained. In cases of multiple births, one baby was selected by lottery method to the study.

\section{Populations}

\section{Source populations}

All postnatal mothers that gave birth to preterm and/or low birth weight babies and their new-borns in Dil Chora and Hiwot Fana Specialized hospitals during the study period.

\section{Study populations}

All postnatal mothers in maternity, NICU and KMC wards who gave preterm and/or low birth weight with their new-borns and that fulfil inclusion criteria.

\section{Inclusion criteria}

Normal post natal mothers having pre term, LBW, Stable infants breathing on their own, and infants without life-threatening disease or malformations that are willing to participate in this study.

\section{Data collection}

Data were collected by face-to-face interview using standardized questionnaire. For practice part, direct observation was done when they position, breast feed and ambulate.

\section{Data quality control}

The data collection tool was prepared in English and translated to local languages of the mothers (Oromiffa, Somali and Amharic). Pretest was conducted in 5\% of mothers in Haramaya hospital. Two days training was given to data collectors by investigators. Close supervision was undertaken during data collection by supervisors and investigators.

\section{Statistical Analysis}

Data were coded, and entered in to Epi Data version 3.02 and exported to SPSS version 20 for analysis. Descriptive statistics was used to describe the frequency, mean and standard deviations. Tables and graphs were used to present the data.

\section{Ethical Clearance}

Ethical clearance was obtained from Haramaya University College of Health and Medical Sciences Institutional Health Research and Ethics Review Committee (IHRERC) and submitted to Harari and Dire Dawa health bureaus. Mothers were informed about the objectives of the study, risks and benefits. Then, participant information was read, written and signed consent was obtained. After data collection health education was given to all postnatal mothers and their families about benefits of kangaroo mother care.

\section{Results}

\section{Socio-demographic characteristics}

A total of 349 postnatal mothers with preterm and low birth weight were participated in this study. Religion of Muslim, Orthodox Christian and protestant constitute $51.24 \%, 38.02 \%$ and $7.44 \%$ of study participants. The mean family's monthly income was 2127 Ethiopian Birr (97 USD). The mean family size was six (Table 1). 
Citation: Roba AA, Binoy S, Naganuri MA (2017) Knowledge, Attitude and Practice of Kangaroo Mother Care by Postnatal Mothers who Gave Birth to Preterm and Low Birth Weight Babies in Public Hospitals, Eastern Ethiopia. J Neonatal Biol 6: 264. doi: $10.4172 / 2167-0897.1000264$

Page 3 of 5

\begin{tabular}{|l|l|l|}
\hline Variables & Frequency & Percentage \\
\hline Maternal age & 29 & 8.31 \\
Less than18 & 320 & 91.69 \\
\hline Maternal education & 174 & 49.86 \\
No education & 87 & 24.93 \\
Primary education (grade 1-8) & 88 & 25.21 \\
Secondary education and above & 17 & 4.87 \\
\hline Maternal occupation & 241 & 69.05 \\
House Wife & 75 & 21.49 \\
Civil servant & 16 & 4.59 \\
Merchant/Non-governmental Organization & & \\
Daily Labourers & 195 & 55.87 \\
\hline Residence & 154 & 44.13 \\
Urban & & \\
Rural & 256 & 73.35 \\
\hline ANC follow-up & 93 & 26.65 \\
Yes & & \\
No & & \\
\hline
\end{tabular}

Table 1: Socio-demographic characteristics of postnatal mothers in Dilchora and HFSH, 2016.

\section{Socio-demographic characteristics of the neonate}

\begin{tabular}{|c|c|c|c|}
\hline \multicolumn{2}{|l|}{ Characteristics } & \multirow{2}{*}{$\begin{array}{l}\text { Frequency } \\
186\end{array}$} & \multirow{2}{*}{$\begin{array}{l}\text { Percentage } \\
53.3\end{array}$} \\
\hline Sex & Male & & \\
\hline & Female & 163 & 46.7 \\
\hline \multirow[t]{2}{*}{ Health status } & Healthy & 198 & 56.73 \\
\hline & Sick & 151 & 43.27 \\
\hline \multirow[t]{3}{*}{ Type of delivery } & SVD & 244 & 69.91 \\
\hline & Instrumental & 38 & 10.88 \\
\hline & $\mathrm{C} / \mathrm{S}$ & 67 & 19.21 \\
\hline \multirow[t]{3}{*}{ Type of Birth } & Singleton & 177 & 50.71 \\
\hline & Twins & 93 & 26.65 \\
\hline & Multiple & 79 & 22.64 \\
\hline \multirow[t]{4}{*}{ Gestational age } & Term & 69 & 19.77 \\
\hline & Moderate to late preterm & 240 & 68.77 \\
\hline & Very preterm & 32 & 9.17 \\
\hline & Extremely preterm & 8 & 2.29 \\
\hline \multirow[t]{3}{*}{ Birth weight } & LBW & 250 & 71.63 \\
\hline & Very LBW & 81 & 23.21 \\
\hline & Extremely LBW & 18 & 5.16 \\
\hline
\end{tabular}

Majority of the neonates included in this study were healthy (56.73\%) and LBW (71.67\%). The mean birth weight was $1820 \mathrm{~g}$, and the mean gestational age was 35.4 weeks (Table 2).

\section{Knowledge of mothers regarding KMC}

All of the study participants heard about kangaroo mother care. Regarding the benefits of kangaroo mother care to the baby, 186 (53.29\%) were responded that KMC mainly helps to maintain the body temperature warm, 58 (16.62\%) for frequent feeding and 105 (30.09\%) didn't know the main use of KMC. Most of the mothers 189 (54.15\%) didn't know the frequency of feeding the baby in KMC position. KMC positioning was correctly described by $116(33.24 \%)$ mothers while the rest $233(66.76 \%)$ were unaware of proper KMC positioning. Concerning length of KMC procedure, 23 (6.59\%) mothers responded it was based on mothers interest, $46(13.18 \%)$ until the baby achieves regular body temperature, $38(10.88 \%)$ until the child matures and the remaining 241 (69.05\%) didn't know for how long KMC intended to be given. Regarding clothing during KMC, 233 (66.76\%) mothers had no information how to cloth the baby, 29 (8.3\%) to dress light clothes only and only $87(24.93 \%)$ correctly stated baby should dress cap, socks and nappy (only with diaper). Most of the respondents 204 (58.45\%) mentioned the main barriers of KMC utilization were due to lack of knowledge regarding KMC practice, 116 (33.24\%) perceived it is not comfortable to the mothers while the remaining 29 (8.31\%) mentioned culturally unacceptable (Figure 1).

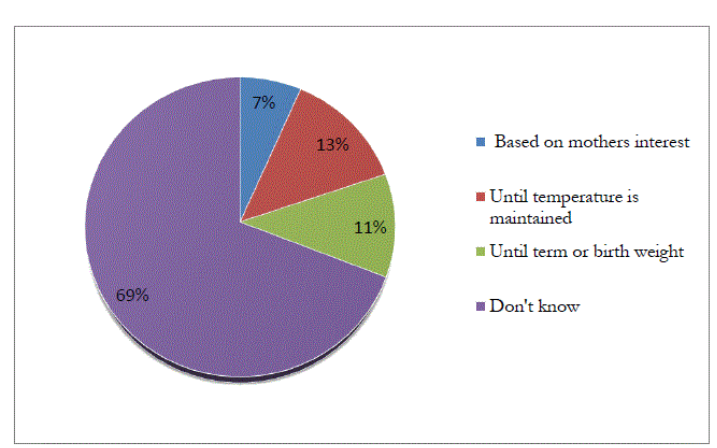

Figure 1: Knowledge of mothers regarding length of KMC for their preterm/low birth weight babies.

\section{Attitude of mothers towards KMC}

The majority of mothers 221 (63.33\%) felt positive regarding implementation of $\mathrm{KMC}$ for it corrects the temperature, increase attachment and improve the growth of their small babies. On the other hand, $195(55.87 \%)$ believed that KMC has positive effect on breast feeding (Figure 2).

Table 2: Characteristics of the neonate among postnatal mothers in Dilchora and HFSH Ethiopia 2016. 
Citation: Roba AA, Binoy S, Naganuri MA (2017) Knowledge, Attitude and Practice of Kangaroo Mother Care by Postnatal Mothers who Gave Birth to Preterm and Low Birth Weight Babies in Public Hospitals, Eastern Ethiopia. J Neonatal Biol 6: 264. doi: $10.4172 / 2167-0897.1000264$

Page 4 of 5

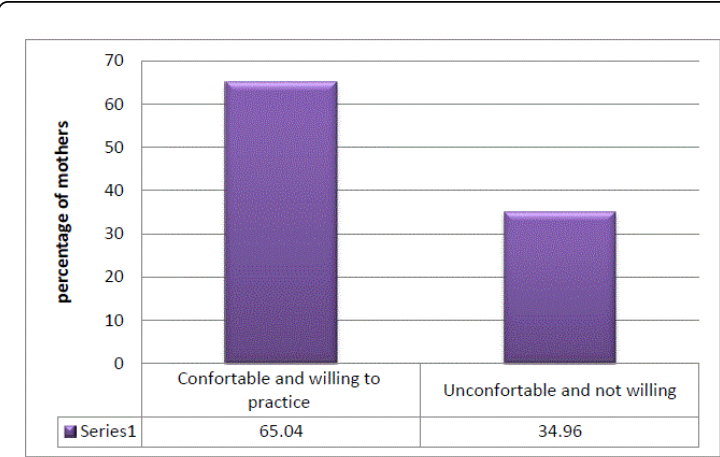

Figure 2: Willingness to practice KMC after getting explanation from health professionals.

\section{Practice of KMC}

During the study period, 189 (54.15\%) mothers practiced KMC in hospitals and also willing to continue at home. Among them, 151 (79.89\%) exclusively breast feed their baby as frequent as possible while $20(10.58 \%)$ feed only when the child cried but the remaining 18 $(9.53 \%)$ used formula milk. The mean duration on KMC was 9 days and $174(76 \%)$ mothers continued KMC for average of two weeks in the hospitals. The mean duration of KMC was $2 \mathrm{~h} /$ day (Figure 3 ).

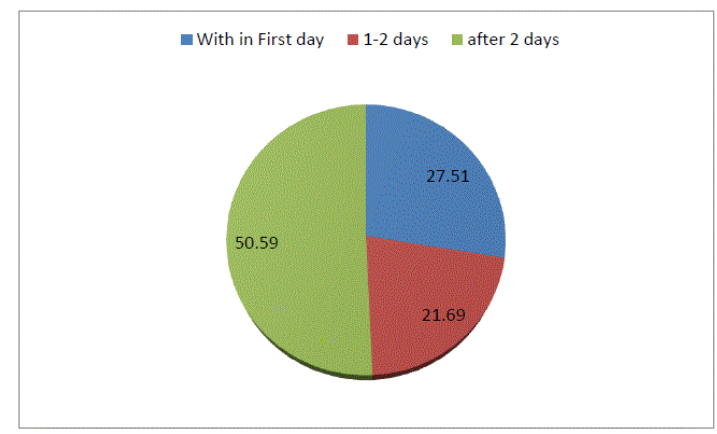

Figure 3: Time of initiation of KMC for preterm and low birth weight neonates

\section{Discussion}

Knowledge of mothers regarding benefits of KMC to their small babies in this was (64\%). This is comparable with a study in Malawi in which mothers had high level of knowledge about the significant benefits of Kangaroo Mother Care even if $84 \%$ were not aware of the services prior to their hospitalisation [13]. Our finding was lower than that of a Ghanaian study in which $90 \%$ of mothers mentioned KMC as beneficial strategy for them and their low birth weight babies [14].

The time of initiation of KMC in this study was comparable with other similar studies. In India, 55.4 percent of the women initiated $\mathrm{KMC}$ within $72 \mathrm{~h}$ of birth [15], in Bangladesh the mean time for initiation of KMC was $1.1 \mathrm{~h}$ [16] but in Ghana $84.6 \%$ started within 24 $\mathrm{h}$ [14]. On the other hand, the mean duration of KMC per day in this study was $2 \mathrm{~h}$. This finding was better than that of $32 \mathrm{~min}$ in a day in Iran [17] and comparable with the findings of India in which the duration of KMC was 1, 2 and $12 \mathrm{~h}$ as felt by $52 \%, 19.6 \%$ and $6.5 \%$ of mothers respectively [18]. When compared to USA finding of 4.47 contact hours per day, our finding was lower by more than a half [19]. These differences may be due to differences in knowledge, attitude and interest of mothers to practice $\mathrm{KMC}$ as well as openness of the KMC guidelines for duration of implementation.

This study showed that 227 (65.04\%) mothers have accepted KMC as a good strategy. This was similar with Odisha, Gujarat and Maharashtra findings [15]. However, the finding was lower than $96 \%$ acceptance of KMC by Indian mothers [20], 87.1\% mothers who had favourable opinion towards the practice of KMC in Ghana; and 96.6\% of mothers feel positive in Cape Town [21].

\section{Conclusion and Recommendations}

The majority of mothers felt positive regarding implementation of KMC. The study also revealed that more than half of the study participants practiced $\mathrm{KMC}$ in hospitals and also willing to continue at home. Therefore, it will be crucial if there are health education sessions during ANC follow-up for complete acceptance of KMC after delivery.

\section{Declarations}

\section{Authors contribution}

All authors contributed their part since proposal writing, training of data collectors, data collection supervision, quality assurance, data analysis and manuscript preparations.

\section{Acknowledgement}

We would like to express our deepest gratitude to Haramaya University, College of Health and Medical College Sciences for success of this study. We would like also to thank Institutional Health Research and Ethics Review Committee for the ethical approval of this study.

\section{References}

1. WHO (2003) Kangaroo mother care: A practical guide, Geneva.

2. UNICEF (2004) Low birth weight: Country, regional and global estimates.

3. Avery ME, Tooley WH, Keller JB (1987) Is chronic lung disease in low birth weight infants preventable? A survey of 8 centers. Pediatrics 79 : 26-30.

4. WHO (2016) Preterm birth. World Health Organization.

5. Trevisanuto D, Putoto G, Pizzol D, Serena T, Manenti F, et al. (2016) Is a woollen cap effective in maintaining normothermia in low-birth-weight infants during kangaroo mother care? Study protocol for a randomized controlled trial. Trials 17: 265.

6. Assefa N, Berhane Y, A. W (2012) A wealth status, mid upper arm circumference (MUAC), and antenatal cares (ANC) are determinants for LBW in Kersa Ethiopia. PLoS ONE 7.

7. Tema T (2006) Prevalence and determinants of low birth weight in Jimma zone, Southwest Ethiopia. East Afr Med J 83.

8. Taddesse KZ (2014) A low birth weight and associated factors among newborns in Gonder town. Indo Glob J Pharm Sci 4: 74-80.

9. Tadesse Alemu, Melaku Umeta (2016) Prevalence and predictors of "small size" babies in Ethiopia: In-depth analysis of the Ethiopian Demographic and Health Survey, 2011. Ethiop J Health Sci 26: 243-250. 
Citation: Roba AA, Binoy S, Naganuri MA (2017) Knowledge, Attitude and Practice of Kangaroo Mother Care by Postnatal Mothers who Gave Birth to Preterm and Low Birth Weight Babies in Public Hospitals, Eastern Ethiopia. J Neonatal Biol 6: 264. doi: $10.4172 / 2167-0897.1000264$

Page 5 of 5

10. Charpak N, Ruiz JG, Zupan J, Cattaneo A, Figueroa Z, et al. (2005) Kangaroo mother care: 25 years after. Acta paediatr 94: 514-522.

11. WHO (2010) World Health Organization: Estimates. State of the World's New-borns. Washington, DC: Saving New-born Lives, Save the Children/ USA, 1-49.

12. http://uofmhealthblogs.org/wp-content/uploads/2013/05/C. 3KangarooCare.pdf

13. Chisenga JZ, Chalanda M, Ngwale M (2015) Kangaroo Mother Care: A review of mothers' experiences at Bwaila hospital and Zomba Central hospital (Malawi). Midwifery 31: 305-315.

14. Nguah SB, Wobil PN, Obeng R, Yakubu A, Kerber KJ, et al. (2011) Perception and practice of Kangaroo Mother Care after discharge from hospital in Kumasi, Ghana: A longitudinal study. BMC Preg Childbirth 11: 99.

15. Rasaily R, Ganguly KK, Roy M, Vani SN, Kharood N, et al. (2017) Community based kangaroo mother care for low birth weight babies: A pilot study. Indian J Med Res 145: 51-57.
16. Pervin J, Gustafsson FE, Moran AC, Roy S, Persson LA, et al. (2015) Implementing Kangaroo mother care in a resource-limited setting in rural Bangladesh. Acta Paediatr 104: 458-465.

17. Namnabati M, Talakoub S, Mohammadizadeh M, Mousaviasl F (2016) The implementation of kangaroo mother care and nurses' perspective of barriers in Iranian' NICUs. Iran J Nurs Midwifery Res 21: 84-88.

18. Muddu GK, Boju SL, Chodavarapu R (2013) Knowledge and awareness about benefits of Kangaroo Mother Care. Indian J Pediatr 80: 799-803.

19. Hake-Brooks SJ, Anderson GC (2008) Kangaroo care and breastfeeding of mother-preterm infant dyads 0-18 months: A randomized, controlled trial. Neonatal Netw 27: 151-159.

20. Parmar VR, Kumar A, Kaur R, Parmar S, Kaur D, et al. (2009) Experience with Kangaroo mother care in a neonatal intensive care unit (NICU) in Chandigarh, India. Indian J Pediatr 76: 25-28.

21. Rosant C (2009) Knowledge of and attitudes towards kangaroo mother care in the eastern sub-district, Cape Town [Mini-thesis]: University of Western Cape, p: 63. 